\title{
The promise and peril of parallel chat in video meetings for work
}

\author{
Advait Sarkar \\ advait@microsoft.com \\ Microsoft Research \\ Cambridge, United Kingdom \\ University of Cambridge \\ Cambridge, United Kingdom

$$
\begin{gathered}
\text { Rachel Bergmann } \\
\text { Sharon Gillett } \\
\text { Danielle Bragg } \\
\text { Nancy Baym } \\
\text { v-raberg@microsoft.com } \\
\text { sharong@microsoft.com } \\
\text { dabragg@microsoft.com } \\
\text { baym@microsoft.com } \\
\text { Microsoft Research } \\
\text { Cambridge, MA, USA }
\end{gathered}
$$

\author{
Sean Rintel \\ Damian Borowiec \\ serintel@microsoft.com \\ v-dborowiec@microsoft.com \\ Microsoft Research \\ Cambridge, United Kingdom \\ Abigail Sellen \\ asellen@microsoft.com \\ Microsoft Research \\ Cambridge, United Kingdom
}

\begin{abstract}
We report the opportunities and challenges of parallel chat in workrelated video meetings, drawing on a study of Microsoft employees' remote meeting experiences during the COVID-19 pandemic.

We find that parallel chat allows groups to communicate flexibly without interrupting the main conversation, coordinate action around shared resources, and also improves inclusivity. On the other hand, parallel chat can also be distracting, overwhelming, and cause information asymmetries.

Further, we find that whether an individual views parallel chat as a net positive in meetings is subject to the complex interactions between meeting type, personal habits, and intentional group practices. We suggest opportunities for tools and practices to capitalise on the strengths of parallel chat and mitigate its weaknesses.
\end{abstract}

\section{CCS CONCEPTS}

- Human-centered computing $\rightarrow$ Empirical studies in collaborative and social computing.

\section{KEYWORDS}

videoconferencing, meetings, parallel chat, cscw, survey, poll, diary, accessibility

(c) Advait Sarkar, Sean Rintel, Damian Borowiec, Rachel Bergmann, Sharon Gillett, Danielle Bragg, Nancy Baym, Abigail Sellen 2021. This is the author's version of the work. It is posted here for your personal use. Not for redistribution. The definitive version was published in $\mathrm{CHI}$ Conference on Human Factors in Computing Systems Extended Abstracts (CHI '21 Extended Abstracts), May 8-13, 2021, Yokohama, Japan ACM, New York, NY, USA, 8 pages. https://doi.org/10.1145/3411763.345179.

CHI '21 Extended Abstracts, May 8-13, 2021, Yokohama, Japan

(C) 2021 Copyright held by the owner/author(s). Publication rights licensed to ACM.

ACM ISBN 978-1-4503-8095-9/21/05 ..\$15.00

https://doi.org/10.1145/3411763.3451793

\section{ACM Reference Format:}

Advait Sarkar, Sean Rintel, Damian Borowiec, Rachel Bergmann, Sharon Gillett, Danielle Bragg, Nancy Baym, and Abigail Sellen. 2021. The promise and peril of parallel chat in video meetings for work. In CHI Conference on Human Factors in Computing Systems Extended Abstracts (CHI '21 Extended Abstracts), May 8-13, 2021, Yokohama, Japan. ACM, New York, NY, USA, 8 pages. https://doi.org/10.1145/3411763.3451793

\section{INTRODUCTION AND BACKGROUND}

Although audio/visual (A/V) modalities dominate remote and hybrid video meetings, most platforms enable attendees to simultaneously post text, images, files, links etc. in a meeting chat area (Figure 1).

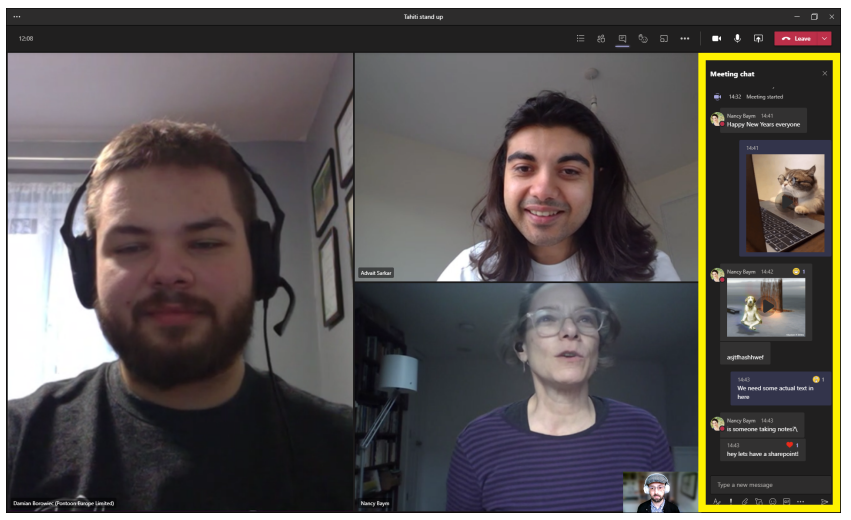

Figure 1: Parallel chat (yellow box) in a video meeting on Microsoft Teams. 
This parallel chat is typically open to all meeting invitees and flows concurrently with the A/V focus of the meeting. The COVID19 pandemic has thrown the use of parallel chat in video meetings into sharp relief, yet it is largely overlooked as an optimisation target for tools and best practices. Meeting platforms provide only brief instructions [61], and even comprehensive remote work guides are largely quiet on the subject [2]. Their design is limited to text panes, windows, or overlays, and while machine learning has enabled live meeting transcription and translation (e.g. [23, 44], it has not yet impacted meeting chat. As parallel chat becomes commonplace, we need to better understand its opportunities and challenges so that we can recommend improvements in practices and design.

\subsection{Prior Work}

Video-mediated collaboration evolved alongside and often with chat in asynchronous and quasi-synchronous workspaces [46], but while research collections of video-mediated communication [18], media spaces [25], and remote work [32] provide frameworks and designs for multi-modal collaboration, there is surprisingly little prior exploration of parallel chat in video meetings. Rather, parallelism is discussed in terms of $\mathrm{A} / \mathrm{V}$ side conversations [10], combining digital and physical resources [9], and enabling non-A/V resources [30]. While task effectiveness, trust, or other values have been compared and contrasted in videoconferencing against other modalities $[8,16,45]$, comparisons of video meetings with and without parallel chat are not available. In work contexts, video-meeting research on attention and multi-tasking has noted parallel chat as one among many distractions [11, 34, 41], but not provided detail.

The most evidence we have comes from educational contexts, in which live online $\mathrm{A} / \mathrm{V}$ of teacher presentations and student-teacher or student-student interactions is accompanied by a chat 'backchannel' $[4,60]$. Its advantages include enabling questions, clarifications, affirmations, and posting resources. Everyone may contribute simultaneously, including less those vocal, which develops community and maintains engagement.

However, parallel chat has the major disadvantage of being distracting. Again, from the educational context, beyond the obvious problem of students having off-topic discussions, even when ontopic there is a danger of "processing information at increasingly superficial levels while [attempting] to juggle tasks and transfer attention across multiple domains simultaneously" [27, 60]. This "continuous partial inattention" [42] may permeate down to the micro level, and effect everyone in the encounter, e.g. teachers using parallel chat to scaffold language teaching via Skype may disattend some student disfluencies [31]. Yardi [60] argues for exploring how scaffolding, permeability, and assisted moderation in backchannels might change educational dynamics, but notes that contextual etiquette(s) will need to evolve to deal with distraction.

The other disadvantage of parallel chat is that it may not be accessible or inclusive. This has at least two strands. First, given the increasingly global work environment, we should no longer assume that all participants can engage equally in single dominant language chat. Manual instant annotation of chat-based brainstorming and decision-making has been found to improve cross-cultural participation [35], but such annotation has not made the jump to parallel chat in video meetings. Second, in the U.S., $26 \%$ of adults have some form of disability [19], and similar figures can be found worldwide. However, work on accessibility of online meetings, work environments, and education [7, 15, 29, 52], and accessibility guidelines $[1,33]$, has not focused on parallel chat.

Livestreaming services incorporate parallel chat for audiences watching content as diverse as video gameplay [24], eating [14], and events [55]. Hosts have learned to permeate their streams with audience engagement techniques in the chat $[13,58]$ [57], but the chat is also rife with abuse and spam that is amplified by imitation, such that hosts have also had to learn how to shape pro and anti-social behavior with moderation and example-setting [53]. Livestreaming services themselves are developing more holistic multi-modal experiences, such as "Danmaku", in which text and reactions float over video [36, 37, 40,59]. Danmaku concepts have not yet made the jump to parallel chat in commercial video meeting systems, with the exception of floating reactions and hand-raising [43]. This may be entirely understandable given that its combination of modalities seems to be a high cognitive load just to comprehend, let alone engage with. However, research on the StreamWiki [38] system has found that, in the context of knowledge sharing live streaming, Danmaku can be combined with other techniques to enable viewers to interactively learn material as well as help producers and moderators produce useful archives of interactive learning experiences for future asynchronous use.

Parallel chat in work video meetings has evolved somewhat under the noses of researchers, and yet there are clearly significant challenges for both practice and design if multi-modal engagement is becoming a professional expectation.

\section{METHOD}

Between mid-April and mid-August 2020, we conducted a large scale study of Microsoft employees' experiences in remote meetings while working from due to COVID-19. To enable global data collection over a significant period of time and covering multiple topics (of which parallel chat was just one), and to provide a rich quantitative and qualitative picture, diaries were used to capture changing reflections on experiences or similar experiences at different times [47], and these were augmented with one-off polls on specific topics to dive more deeply into specific topics [5]. Recruited via internal mailing lists between April and June, 849 participants completed the onboarding study, 715 completed at least one diary entry, and 357 at least one poll. For this report we draw from the onboarding survey, a poll on parallel chat, and relevant diary entries. Full methodology and participation details are available in a technical report [49].

Our parallel chat poll received 149 responses. Participants answered six questions about their use and experience of parallel chat using a 7-point Likert scale (Strongly Disagree - Strongly Agree). The questions and the breakdown of responses can be seen in Figure 2. The poll ended with the free text prompt: "What experience/s led to your answers above? For example: Does chat become more or less distracting or useful depending on the type of meeting, or the meeting size? Are there different kinds of chat during meetings? Do you feel obligated to use text chat in meetings?" We included example prompts for the free text response to encourage participants to explain the reasoning for their Likert scale responses. 
Participants could also author up to 24 diary entries, organized in three cycles of eight guided topics: Physical workspace, Interaction, Productivity, Tools, Multitasking, Types of meetings, Time in meetings, and Approaches to meetings . In these entries, participants occasionally mentioned parallel chat experiences without respect to a specific question as they did in the poll. In total 159 unique participants mentioned 331 parallel chat issues in the diaries.

A key linked participants to onboarding, diary, and poll data. Verbatims were scrubbed for all identifying referents. For qualitative analysis of the verbatims we used semantic thematic analysis [6] to group responses representing how participants used parallel chat and their evaluations of its effectiveness. One researcher coded the poll verbatims and another coded the diary verbatims (both to saturation) and then the team aggregated themes [20]. Throughout the paper we indicate in parentheses the number of individuals who mentioned a theme at least once.

\section{RESULTS}

Parallel chat in video meetings was common for a substantial majority of participants. From the onboarding survey $(\mathrm{N}=849), 69.7 \%$ reported using parallel chat. Of the total, $26.6 \%$ reported using parallel chat in every meeting or almost every meeting, $24.1 \%$ at least once a week, $16.8 \%$ a few times a month, and $2.1 \%$ once a month or less.

In our poll ( $\mathrm{N}=149)$ (Figure 2), most respondents reported an increase in parallel chat use after the shift to remote work. Respondents were polarized over whether parallel chat was distracting. However, they were positive about the value of chat for helping with conversational issues and adding resources, and most felt it was a net positive.

P3: [... I very frequently use text chat to share links, screenshots, etc. that are relevant to the discussion, as well as quick thoughts signals of assent with the speaker, if I don't want to interrupt the main thread. On the whole, I find the ability to have concurrent chat very helpful for effective meetings, even if it can be a distraction at times.

P154: [...] there have been meetings where important links were able to be provided in the text chat, important and "relevant ${ }^{*}$ topics were brought up and then incorporated into the meeting, etc - these are times when I feel like I really could not live without [it][...].

Most respondents found that use of parallel chat had increased for both themselves (72\%) and others (76\%) since mandatory working from home (aggregating "Somewhat Agree", "Agree", and "Strongly Agree"). This is likely due to increased remote meetings [3] and increased appreciation for the uses of parallel chat.

Participants in both the poll and diaries reported using parallel chat for at least seven distinct types of messages:

- Questions for the speaker or someone else in the meeting (96 participants)

- Links to resources such as documents and webpages (64 participants)

- Unrelated conversation held in the same chat (44 participants).

- Voicing agreement with the speaker, or sending messages of praise/congratulations ('kudos'). (40 participants)

- Adding information to what is being said, or starting a conversation about a related topic (37 participants)
- Responses to previous messages (34 participants)

- Humour and casual conversation (26 participants)

\subsection{Positive impacts of parallel chat}

3.1.1 Inclusion and managing the flow of the primary conversation. A key advantage of parallel chat is participation without interrupting the flow of the A/V conversation (62 participants). Being able to ask a question or make a comment in parallel chat may reduce the competition for the floor $[12,17]$ as defined by the A/V stage, because there is another space in which to have their say. With less competition, there may be in turn, fewer moments of the stop-start competitive overlaps [21] which occur due to latency [48, 50,51, 54] and constrained visual cues [9, 26, 39].

P670: [...] in person, there are visual cues a person wants to speak a hand raised, a lean forward, a clearing of the throat. We don't have those cues in video meetings [...] [It is an] ever-more-important way, especially as meetings get larger [...].

P217: [...] I've found it most useful when we are on a tight schedule and there are several speakers. Once I'm done presenting, and have handed off to another speaker, I usually get on chat to answer all the questions that arose - this helps manage time better \& helps me provide links to answers where necessary, which would benefit everyone.

P349: [...] It has also been wonderful when doing demos, because customers can ask questions (and we often answer them) in text chat vs. interrupting the demo. [...]

Moreover, parallel chat gives participants a way to engage if they are otherwise unable to get a chance to speak. Even though Microsoft Teams and other systems now include hand-raise features that mark a participant as desiring a turn, these may go unnoticed or there may simply be too many to accommodate. Parallel chat offers a way to have a say while avoiding the risk of a hand-raise not being seen. Further, since a hand raise is a contextless bid for the floor, asking a question or a comment in the parallel chat enables a method for speakers or moderators to triage potential engagement. In this way, parallel chat may make meetings more inclusive (41 participants).

P208: Text chat can be a great way for more introverted members of staff to contribute to a conversation. [...]

P640: [...] It also allows users who have distracting home lives to participate without fear of judgment. [...]

P153: [...] people contributing through chat that might not have a voice otherwise - either limited by technology (no microphone), environment (loud, distracting) or personal preference (shy, new, still finding the way in the team's culture.)

P222: [...] It helps level the "playing field" by allowing all participants to have a voice and engage by sharing ideas and opinions, [...] it's one of the most valuable meeting capabilities [...]

3.1.2 Coordination of action and collaboration. Another key function of parallel chat is to share links to relevant resources and documents (64 participants). Many participants noted that such sharing might have otherwise been follow-up actions. Moreover, as some platforms enable parallel chat to persist beyond the end of the A/V meeting, it can act as both a record and a means of enabling post-meeting discussion (34 participants).

P12: [...] individuals will reference external materials, items, specs, etc and when they include a link to the referenced material it provides 


\begin{tabular}{|c|c|c|c|c|c|c|c|c|}
\hline Prompt & $\begin{array}{l}\text { Strongly } \\
\text { Disagree }\end{array}$ & Disagree & $\begin{array}{l}\text { Somewhat } \\
\text { Disagree }\end{array}$ & Neutral & $\begin{array}{l}\text { Somewhat } \\
\text { Agree }\end{array}$ & Agree & $\begin{array}{l}\text { Strongly } \\
\text { Agree }\end{array}$ & Sparkline \\
\hline $\begin{array}{l}\text { My use of text chat in meetings has increased compared to } \\
\text { before mandatory working from home. }\end{array}$ & $2 \%$ & $7 \%$ & $5 \%$ & $14 \%$ & $21 \%$ & $24 \%$ & $26 \%$ & \\
\hline $\begin{array}{l}\text { Other people's use of text chat in meetings has increased } \\
\text { compared to before mandatory working from home. }\end{array}$ & $1 \%$ & $5 \%$ & $2 \%$ & $17 \%$ & $19 \%$ & $28 \%$ & $29 \%$ & \\
\hline $\begin{array}{l}\text { I find text chat in meetings distracting from the } \\
\text { presentation or discussion. }\end{array}$ & $7 \%$ & $24 \%$ & $13 \%$ & $11 \%$ & $28 \%$ & $10 \%$ & $13 \%$ & 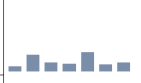 \\
\hline $\begin{array}{l}\text { Text chat in meetings helps overcome conversational } \\
\text { issues like interruption. }\end{array}$ & $1 \%$ & $5 \%$ & $3 \%$ & $7 \%$ & $23 \%$ & $36 \%$ & $25 \%$ & \\
\hline Text chat in meetings helps add more resources like links. & $0 \%$ & $0 \%$ & $1 \%$ & $1 \%$ & $8 \%$ & $32 \%$ & $57 \%$ & \\
\hline Text chat is a net positive for meetings. & $0 \%$ & $1 \%$ & $3 \%$ & $9 \%$ & $14 \%$ & $42 \%$ & $30 \%$ & \\
\hline
\end{tabular}

Figure 2: Parallel chat poll $(\mathrm{N}=149)$ results for all six Likert questions.

clarity that has made a huge impact on my comprehension - especially in areas that are new to me.

P584: [...] it has the nice side effect of having a record of those resources shared or links provided in meetings. [...]

P168: [...] Being able to add documentation, links and relevant information during a meeting and starting a chat that can continue post meeting has really helped cut down on follow ups.

Parallel chat also enables coordination pathways in the face of technical issues such as poor connectivity, device/software malfunctions, camera/microphone issues, etc. (21 participants), coping with language barriers, and written precision when it is useful (e.g. technical terms).

P47: [...][When] one of the participants are on their phone and can't see the presentation[...] we end up sending a screenshot of the current presented screen and the person on the phone can quickly check the chat. [...]

P658: [...] useful to convey information that is hard to convey orally: links, names or contact info, sometimes images, etc.

P260: Usually text chat is relevant links and spellings for technical words, so it has been a great benefit.

P97: [...] It helps if you want to ask question but broadband is poor or there is noise at your end.

3.1.3 Social connection. Casual conversation and humour can give meetings a greater sense of social support and connection, making them more interpersonal and pleasurable (26 participants).

P642: [...] chat provides me an easy way to interact with meeting participants, creating a feeling of participation and providing a sense of the general mood [...] it makes the meetings more personal.

P42: [...] we use text chat to send 'cheers' and fun gifs to celebrate moments [...] this tends to generate a lot of enthusiasm and makes these types of meetings more fun. like people's personalities coming out. [...]

P173: [...] a place where people can express themselves casually, or offer support to the presenter. [...]

\subsection{Negative impacts of parallel chat}

3.2.1 Distraction and division of attention. Parallel chat provides room for unrelated topics to emerge, distracting meeting participants who wish to focus on the meeting topic (68 participants). Participants may feel obliged to divide their attention between the $\mathrm{A} / \mathrm{V}$ and the parallel chat, and many report this division to be difficult to maintain (43 participants).

P579: It's very distracting in large meetings and often is off topic. There are separate conversations occurring between a small few people amongst themselves.

P692: [...] A large meeting where the text chat is busy with a constant stream of loosely related comments [...] If I paid attention to text chat and tried to keep up with it, I would no longer be paying attention to the meeting itself [...].

P245: [...] sometimes it's very distracting as multiple threads are happening that get tangential from the main presenter/speaker. [...] it's really hard to keep track of multiple conversations AND pay attention to the speaker.

3.2.2 Differing expectations on how chat should be used. Informality and side conversations were perceived negatively by some participants (25 participants), with some reporting difficulty finding important information in chat due to message volume or topic irrelevancy (16 participants). Some expressed a desire for more concrete norms and expectations around parallel chat use (15 participants). Others reported having designated moderators, whether to ensure professionalism and respectful behaviour, or to monitor the flow of the meeting and ensure voices are heard (43 participants).

P305: Unless it's stated up front and managed well during the meeting, putting something in the chat window still stops the presentation, everyone stops to read or (in most cases) someone who didn't text in the chat window calls out that the meeting needs to pause as someone else has a question. It still causes a distraction.

P779: [...] Value really depends on call / how participants are using it: in some cases it provides great value, in other cases people use it to socialize, joke, where it can be more distraction than value. [...]

P14: It feels increasingly important to monitor meeting chats alongside the AV component-especially in large meetings. People contribute many kinds of comments [...] 
3.2.3 Information asymmetries. Meeting presenters reported that it was hard to engage with the parallel chat, both in terms of attentional effort and limitations of the platform's interface (27 participants). Further, without timestamps linked to A/V, references to parallel chat in meeting recordings were difficult to follow posthoc, and conversely, visiting the chat afterwards without the A/V context could be confusing (18 participants).

P159: [...] it would be useful for the presenter to see the chat when presenting. A function to highlight questions as opposed to links or comments would be great, too.

P153: [...] I have to keep an eye on the text chat as a presenter, and address points raised / questions asked. But sometimes there is so much chatter of agreement / memes / personal anecdotes that it is hard to find the more important messages. [...]

P350: [...] as a presenter it is almost impossible to keep up with chat and present coherently. [...] Additionally, for recorded meetings, [...][it is hard when people] refer to something in the chat that the listener has no context for.

\subsection{Factors affecting parallel chat use and outcomes}

3.3.1 Demographic factors. We found no relationships with meaningful effect sizes between participants' chat use and their job role (e.g., engineering, research, sales, etc.) or prior work from home status. However, as noted above, women in the age group 25-34 were more likely to strongly agree that their chat use has increased, in comparison to any other gender-age group (Figure 3) (59\% of women aged $25-34$, versus $27 \%$ across all well-represented groups in our sample; $Z=2.9924, p=0.00278$ ).

\begin{tabular}{l|ll} 
Age & Men & Women \\
\hline $25-34$ & $20 \%$ & $59 \%$ \\
$35-44$ & $8 \%$ & $29 \%$ \\
$45-64$ & $24 \%$ & $26 \%$
\end{tabular}

Figure 3: Increased chat use was most reported by women aged 25-34. The figure shows the proportion responding 'strongly agree' that their own chat use has increased. Age groups below 25/above 64 omitted due to low sample size.

A similar finding was reflected in the relative frequency of themes in the combined journal and poll verbatims: women were twice as likely as men to report using parallel chat for questions and answers during meetings (16\% of women, versus $8 \%$ of men; $Z=3.3998, p=0.00068$ ). This may be because women, particularly younger women, find it difficult to be heard in meetings, which is consistent with research on gender and meeting participation [28].

3.3.2 Meeting type and team dynamics. Several participants observed that chat can be both beneficial and distracting, depending on the nature of the meeting and its participants. Major distinctions were drawn depending on the size of the group, whether the meeting was in the form of a talk/presentation, and how familiar the participants were with each other. The likelihood of distraction was greater both in large meetings and presentations. However, some participants felt the opposite: that the use of informal messages made a large group discussion feel more community-minded, inclusive, and energetic. Participants were more conservative with their chat use when the audience was unfamiliar, but within the context of a regular team meeting, participants developed norms around chat use, whether explicit or unspoken.

P267: I'm torn - it's satisfying in some ways to be able to chime in and interact - we give each other a lot of hearts on my team, but it totally pulls away from the presentations.

P252: Distractions are less about the use of chat and more about the people involved. Sometimes there is conversation amongst participants that doesn't directly align with the content - other times it stays somewhat quiet. I think it is starting to mirror interactions people have in gatherings of different types and sizes in the analog world. [...]

P312: On my team, we have meetings that are heavy on chat. Chat is particularly effective as it serializes communication, however like anything, if overused it becomes a distraction. [...]

\section{IMPLICATIONS FOR BEST PRACTICES AND DESIGN}

\subsection{Guidelines for best practices}

The polarization around parallel chat indicates the need for all attendees to be more intentional in balancing utility and distraction. Further, several of our participants reported that moderators improve the effective use of parallel chat. We suggest the following guidelines to make parallel chat more effective. Meeting organisers, teams, and moderators should make a guideline document (e.g. [22]) available generally and at the beginning of meetings.

Establish expectations: Share guidance on expected uses of chat before the meeting starts. For example, if a meeting features a speaker, announce their preference up front for when and how to receive questions. Because monitoring and facilitating chat imposes cognitive load, plan to rotate moderator duties across meetings and within longer meetings.

Consider accessibility: Chat-related accessibility challenges include processing parallel sources in multiple modalities (e.g. with a sensory disability), consuming and generating text (e.g. with reading disabilities), and understanding sentiment behind text (e.g. with autism). Ensure that accessibility requests are met, for example by providing text descriptions of non-text chat content and visual content in a video call (e.g. for participants with visual impairments), and leaving appropriate time for participants with disabilities to respond (e.g. if using an interpreter or screen reader).

Encourage engagement: Encourage chat that explores different aspects of the meeting's topic, for example by providing supporting links or materials, questions, or requests for clarifications. Also encourage chat that allows more voices to be heard, such as contributions from people who may otherwise have difficulty getting heard for many reasons, including lack of seniority/power, minority status, or disability. Moderators can encourage positive types of chat by speaking them aloud or asking their creators to voice their written content if desired. 
Discourage distraction: Discourage chat that diverges from the meeting's topic, is of interest to only a small subgroup, or is inaccessible to those requiring accommodation. Diplomatic discouragement may use a private backchannel to avoid public shaming, or by asking participants engaged in a divergent topic to shift to a separate channel.

Loop in the speaker: Communicate non-intrusively with the current speaker to facilitate key information exchange with the audience. In particular, monitor the chat for questions that may be asynchronously directed to the speaker, and raise them in a flowrespecting manner.

Synthesize and disseminate: Incorporate chat highlights into meeting recaps. Example highlights from chat could include relevant links or topics raised that warrant further attention or discussion.

\subsection{Implications for design}

Differentiate and annotate the content of chat: Despite there being several distinct categories of chat messages, current tools do not visually distinguish them. This makes it hard for presenters and audience members to visually search text for the material most relevant to them. While manual annotation [35] is a good first step, automated classification of messages could vastly reduce the cognitive burden by differentiating different kinds of material (both visually for sighted users, and through other mechanisms for blind and low vision users). For example, this would enable kudos (e.g. positive emoji, terms like "great job") to be visually differentiated from substantive comments. Questions, clarifications, comments, kudos, on- and off- topic talk could potentially all be differentiated. Further, automated categorisation could enable semantic zooming [56] in chat, where zooming out would group posts by time, keyword, reaction, etc., to reveal patterns and 'hotspots' of activity and enable effective scanning.

Integrate chat with $A / V$ : Given the problem of attending to separated $\mathrm{A} / \mathrm{V}$ and chat, there are lessons to be learned from Danmaku [36, 37, 40,59] and even recent film and television in which text and other resources form an integrated narrative. For example, nontextual activity indicators to enable presenters to see when chat is busy or quiet, intelligent placement of chat to avoid other text or attach chat to parts of the visual image, highlighting messages with posted terms that match spoken terms, and creating non-intrusive question queues that show and hide questions automatically for presenters as relevant or addressed. These features complement differentiated chat. For example, kudos from chat could be shown on the $\mathrm{A} / \mathrm{V}$ in disaggregated manner during the presentation but available aggregated afterwards. Images, documents, or websites shared into chat could be elevated to the main $\mathrm{A} / \mathrm{V}$ in a seamless manner.

Connect timestamped textual material: During the meeting, enabling parallel chat, transcript, and meeting notes to be accessed side-by-side could reduce duplication and facilitate more targeted use of each. For improved contextualisation after meetings, timestamping of chat, transcription, and notes with $\mathrm{A} / \mathrm{V}$ would enable later viewers to understand the relationship between them.

Moderator's view: In line with the value of moderators, moderators could have access to special versions of the tools above, and more, so that they could both publicly and privately help manage the manner in which parallel chat integrates with the meeting. This could include the ability to compartmentalise chat content, remove/mute participants, hide content, etc. It could also facilitate non-disruptive information exchange between moderator and presenter.

\section{LIMITATIONS}

Most employees reported on experiences in Microsoft Teams. While this clearly impacts our results, and future research certainly should expand to both other companies and other platforms, the current similarity of chat features in major platforms leads us to believe that this study provides at least a strong high level overview of the phenomena. Our overview shows a range of positive and negative expectations surrounding the use of parallel chat in work meetings, but we do not have sufficient data to explain whether these different expectations were due to individual differences or because of different tasks/types of collaboration. This will be a crucial step for future research.

\section{CONCLUSIONS}

Our study has uncovered a diversity of uses and benefits of parallel chat in work video meetings. It allows for communication during a meeting, enables effective coordination around resources, acts as a record and a venue for continued discussion, enables pathways to recovery from technical issues, and makes meetings more inclusive. Parallel chat also has pitfalls. When the chat conversation diverges from the main audio-visual conversation, the chat may distract listeners and cause them to miss out on the primary content. Divergent chats may be missed entirely, distract and derail the primary speaker, or be seen as unprofessional. More intentional use, and especially moderation, may help guide participants to realize its value and avoid its problems. Moreover, the design of the parallel chat experience could respond to these challenges by enabling differentiated and integrated usage. As with many aspects of remote and hybrid meeting practice and design, we argue that there is a need to take a more intentional stance so that organizational purpose is more accountable in the tools we have to achieve it. Any design or normative solutions must recognize the dialectical nature of parallel chat as offering both opportunities and challenges and see the goal as striking the right balances between them.

\section{ACKNOWLEDGEMENTS}

The authors thank the participants for their valuable aggregate contribution, Priscilla Wong for her logistics assistance, and John Tang for initial feedback. We also thank the Associate Chair and reviewers for their effort and suggestions. 


\section{REFERENCES}

[1] AccessSIGCHI. 2020. Accessible Remote Attendance. https://accesssigchi.com/ accessible-remote-attendance/

[2] Atlassian. 2021. Work Life by Atlassian. https://www.atlassian.com/blog

[3] Emily Bary. 2021. Zoom, Microsoft Teams usage are rocketing during coronavirus pandemic, new data show. https://www.marketwatch.com/story/zoommicrosoft-cloud-usage-are-rocketing-during-coronavirus-pandemic-newdata-show-2020-03-30 Section: Industries.

[4] Sharla Berry. 2019. The Role of Video and Text Chat in a Virtual Classroom: How Technology Impacts Community. In Educational Technology and Resources for Synchronous Learning in Higher Education, Jiyoon Yoon and Peggy Semingson (Eds.). IGI Global, Hershey, PA, USA, 173-187. https://doi.org/10.4018/978-15225-7567-2.ch009

[5] Ann Blandford, Dominic Furniss, and Stephann Makri. 2016. Qualitative HCI Research: Going Behind the Scenes. Synthesis Lectures on HumanCentered Informatics 9, 1 (April 2016), 1-115. https://doi.org/10.2200/ S00706ED1V01Y201602HCI034

[6] Virginia Braun and Victoria Clarke. 2006. Using thematic analysis in psychology. Qualitative Research in Psychology 3, 2 (Jan. 2006), 77-101. https://doi.org/10. 1191/1478088706qp063oa

[7] Paula J Burdette, Diana L Greer, and Kari L Woods. 2013. K-12 Online Learning and Students with Disabilities: Perspectives from State Special Education Directors. fournal of asynchronous learning networks 17, 3 (2013), 65-72.

[8] Judee K. Burgoon, Joseph A. Bonito, Bjorn Bengtsson, Artemio Ramirez Jr, Norah E. Dunbar, and Nathan Miczo. 1999. Testing the interactivity model: Communication processes, partner assessments, and the quality of collaborative work. fournal of management information systems 16, 3 (1999), 33-56. ISBN: 0742-1222 Publisher: Taylor \& Francis.

[9] Bill Buxton. 2009. Mediaspace - Meaningspace - Meetingspace. In Media Space $20+$ Years of Mediated Life, Steve Harrison (Ed.). Springer, London, 217-231. https://doi.org/10.1007/978-1-84882-483-6_13

[10] William A.S. Buxton, Abigail J. Sellen, and Michael C. Sheasby. 1997. Interfaces for Multiparty Videoconferencing. In Video-Mediated Communication, Kathleen E. Finn, Abigail J. Sellen, and Sylvia B. Wilbur (Eds.). Lawrence Erlbaum Associates, Mahwah, NJ, 385-400.

[11] Hancheng Cao, Chia-Jung Lee, Shamsi Iqbal, Mary Czerwinski, Priscilla Wong, Sean Rintel, Brent Hecht, Jaime Teevan, and Longqi Yang. 2021. Large Scale Analysis of Multitasking Behavior During Remote Meetings. In Proceedings of the 2021 CHI Conference on Human Factors in Computing Systems (CHI '21). Association for Computing Machinery, New York, NY, USA. https://doi.org/10.1145/3411764. 3445243 event-place: Yokohama, Japan.

[12] Joseph N. Cappella, A. W. Siegman, and S. Feldstein. 1985. Controlling the floor in conversation. In Multichannel integrations of nonverbal behavior. Psychology Press, Hove, UK, 69-103.

[13] Xinyue Chen, Si Chen, Xu Wang, and Yun Huang. 2021. "I was afraid, but now I enjoy being a streamer!": Understanding the Challenges and Prospects of Using Live Streaming for Online Education. Proceedings of the ACM on Human-Computer Interaction 4, CSCW3 (Jan. 2021), 1-32. https://doi.org/10.1145/3432936

[14] Hanwool Choe. 2019. Eating together multimodally: Collaborative eating in mukbang, a Korean livestream of eating. Language in Society 48, 2 (April 2019), 171-208. https://doi.org/10.1017/S0047404518001355

[15] Norman Coombs. 2010. Making online teaching accessible: Inclusive course design for students with disabilities. Vol. 17. John Wiley \& Sons.

[16] Richard L. Daft and Robert H. Lengel. 1986. Organizational information requirements, media richness and structural design. Management science 32, 5 (1986), 554-571. ISBN: 0025-1909 Publisher: INFORMS.

[17] Carole Edelsky. 1981. Who's Got the Floor? Language in Society 10, 3 (1981), 383-421. https://www.jstor.org/stable/4167262 Publisher: Cambridge University Press.

[18] Kathleen E. Finn, Abigail J. Sellen, and Sylvia B. Wilbur (Eds.). 1997. VideoMediated Communication. Lawrence Erlbaum Associates, Mahwah, NJ.

[19] Centers for Disease Control and Prevention. 2020. Disability Impacts All of Us. https://www.cdc.gov/ncbddd/disabilityandhealth/infographic-disabilityimpacts-all.html

[20] Sheila M Fram. 2013. The Constant Comparative Analysis Method Outside of Grounded Theory. (2013), 25.

[21] Janan Ganesh. 2020. Zoom and the lost art of interruption. https://www.ft.com/ content/5745fc60-b0db-4958-bdf4-3bb6307e190d

[22] Sharon Gillett, Danielle Bragg, Advait Sarkar, and Sean Rintel. 2021. Parallel Meeting Chat Guide for Moderators and Participants: Drawing on findings from Microsoft Employees During COVID-19. Technical Report 2021-02-FOW-SIM3. Microsoft Research. https://www.microsoft.com/en-us/research/publication/ parallel-meeting-chat-guide-for-moderators-and-participants/

[23] Google. 2021. Use captions in a video call. https://support.google.com/meet/ answer 9300310

[24] William A. Hamilton, Oliver Garretson, and Andruid Kerne. 2014. Streaming on twitch: fostering participatory communities of play within live mixed media. In
Proceedings of the SIGCHI Conference on Human Factors in Computing Systems. ACM, Toronto Ontario Canada, 1315-1324. https://doi.org/10.1145/2556288. 2557048

[25] Steve Harrison. 2009. Media Space 20+ Years of Mediated Life (1st ed.). Springer Publishing Company, Incorporated.

[26] Christian Heath and Paul Luff. 1993. Disembodied conduct: Interactional asymmetries in video-mediated communication. Technology in working order: Studies of work, interaction, and technology (1993), 35-54. Publisher: Citeseer.

[27] Helene Hembrooke and Geri Gay. 2003. The laptop and the lecture: The effects of multitasking in learning environments. fournal of computing in higher education 15, 1 (2003), 46-64. ISBN: 1042-1726 Publisher: Springer.

[28] Hemshorn de Sánchez Clara S. and Meinecke Annika L. 2020. Social Influence in Meetings: A Gender Perspective. In Managing Meetings in Organizations, Annika L. Meinecke, Joseph A. Allen, and Nale Lehmann-Willenbrock (Eds.). Research on Managing Groups and Teams, Vol. 20. Emerald Publishing Limited, 113-142. https://doi.org/10.1108/S1534-085620200000020006

[29] Marion Hersh, Barbara Leporini, and Marina Buzzi. 2020. Accessibility Evaluation of Video Conferencing Tools to Support Disabled People in Distance Teaching, Meetings and other Activities. In ICCHP. 133.

[30] Ellen A. Isaacs and John C. Tang. 1997. Studying video-based collaboration in context: From small workgroups to large organizations. In Video-mediated communication, Kathleen E. Finn, Abigail J. Sellen, and Sylvia B. Wilbur (Eds.). 173-197. Publisher: New Jersey: Lawrence Erlbaum.

[31] Olga Kozar. 2016. Text Chat During Video/Audio Conferencing Lessons: Scaffolding or Getting in the Way? CALICO fournal 33, 2 (Feb. 2016), 231-259. https://doi.org/10.1558/cj.v33i2.26026

[32] Robert E. Kraut, Jolene Galegher, and Carmen Egido (Eds.). 2014. Intellectual Teamwork : Social and Technological Foundations of Cooperative Work. Psychology Press. https://doi.org/10.4324/9781315807645

[33] Raja S Kushalnagar and Christian Vogler. 2020. Teleconference Accessibility and Guidelines for Deaf and Hard of Hearing Users. In The 22nd International ACM SIGACCESS Conference on Computers and Accessibility. 1-6.

[34] Anastasia Kuzminykh and Sean Rintel. 2020. Classification of Functional Attention in Video Meetings. In Proceedings of the 2020 CHI Conference on Human Factors in Computing Systems (CHI '20). Association for Computing Machinery, New York, NY, USA, 1-13. https://doi.org/10.1145/3313831.3376546 event-place: Honolulu, HI, USA.

[35] Na Li and Mary Beth Rosson. 2014. Using annotations in online group chats. In Proceedings of the SIGCHI Conference on Human Factors in Computing Systems (CHI '14). Association for Computing Machinery, New York, NY, USA, 863-866. https://doi.org/10.1145/2556288.2557209

[36] Lili Liu, Ayoung Suh, and Christian Wagner. 2016. Watching online videos interactively: the impact of media capabilities in Chinese Danmaku video sites. Chinese Journal of Communication 9, 3 (July 2016), 283-303. https://doi.org/10. 1080/17544750.2016.1202853

[37] Zhicong Lu, Michelle Annett, and Daniel Wigdor. 2019. Vicariously Experiencing it all Without Going Outside: A Study of Outdoor Livestreaming in China. Proceedings of the ACM on Human-Computer Interaction 3, CSCW (Nov. 2019), 1-28. https://doi.org/10.1145/3359127

[38] Zhicong Lu, Seongkook Heo, and Daniel J. Wigdor. 2018. StreamWiki: Enabling Viewers of Knowledge Sharing Live Streams to Collaboratively Generate Archival Documentation for Effective In-Stream and Post Hoc Learning. Proceedings of the ACM on Human-Computer Interaction 2, CSCW (Nov. 2018), 1-26. https: //doi.org/10.1145/3274381

[39] Paul Luff, Christian Heath, Naomi Yamashita, Hideaki Kuzuoka, and Marina Jirotka. 2016. Embedded reference: translocating gestures in video-mediated interaction. Research on Language and Social Interaction 49, 4 (2016), 342-361. ISBN: 0835-1813 Publisher: Taylor \& Francis.

[40] Xiaojuan Ma and Nan Cao. 2017. Video-based Evanescent, Anonymous, Asynchronous Social Interaction: Motivation and Adaption to Medium. In Proceedings of the 2017 ACM Conference on Computer Supported Cooperative Work and Social Computing. ACM, Portland Oregon USA, 770-782. https://doi.org/10.1145/ 2998181.2998256

[41] Jennifer Marlow, Eveline van Everdingen, and Daniel Avrahami. 2016. Taking Notes or Playing Games?: Understanding Multitasking in Video Communication. In Proceedings of the 19th ACM Conference on Computer-Supported Cooperative Work \& Social Computing. ACM, San Francisco California USA, 1726-1737. https: //doi.org/10.1145/2818048.2819975

[42] Joseph F McCarthy. 2005. Digital Backchannels in Shared Physical Spaces: Experiences at an Academic Conference. (2005), 4

[43] Microsoft. 2021. Express yourself in Teams meetings with live reactions. https://support.microsoft.com/en-gb/office/express-yourself-in-teamsmeetings-with-live-reactions-a8323a40-3d07-4129-934b-305370a36e21

[44] Microsoft. 2021. View live transcription in a Teams meeting. https://support.microsoft.com/en-gb/office/view-live-transcription-in-ateams-meeting-7a1401ec-73b4-431d-875a-8b6af82b3e15

[45] Robert B. Ochsman and Alphonse Chapanis. 1974. The effects of 10 communication modes on the behavior of teams during co-operative problem-solving. 
International fournal of Man-Machine Studies 6, 5 (1974), 579-619. ISBN: 00207373 Publisher: Elsevier

[46] Judith S. Olson and Gary M. Olson. 2013. Working Together Apart: Collaboration over the Internet. Synthesis Lectures on Human-Centered Informatics 6, 5 (Nov. 2013), 1-151. https://doi.org/10.2200/S00542ED1V01Y201310HCI020 Publisher: Morgan \& Claypool Publishers.

[47] John Rieman. 1993. The diary study: a workplace-oriented research tool to guide laboratory efforts. In Proceedings of the SIGCHI conference on Human factors in computing systems - CHI '93. ACM Press, Amsterdam, The Netherlands, 321-326. https://doi.org/10.1145/169059.169255

[48] Sean Rintel. 2013. Video calling in long-distance relationships: The opportunistic use of audio/video distortions as a relational resource. The Electronic fournal of Communication/La Revue Electronic de Communication (EFC/REC) 23 (2013)

[49] Sean Rintel, Priscilla Wong, Advait Sarkar, and Abigail Sellen. 2020. Methodology and Participation for 2020 Diary Study of Microsoft Employees Experiences in Remote Meetings During COVID-19. Technical Report 2020-10-FOW-SIM1. Microsoft Research. https://www.microsoft.com/en-us/research/publication/methodologyand-participation-for-2020-diary-study-of-microsoft-employees-experiencesin-remote-meetings-during-covid-19/

[50] Karen Ruhleder and Brigitte Jordan. 2001. Co-Constructing Non-Mutual Realities: Delay-Generated Trouble in Distributed Interaction. Comput. Supported Coop. Work 10, 1 (Jan. 2001), 113-138. https://doi.org/10.1023/A:1011243905593 Place: USA Publisher: Kluwer Academic Publishers.

[51] Katrin Schoenenberg, Alexander Raake, and Judith Koeppe. 2014. Why are you so slow?-Misattribution of transmission delay to attributes of the conversation partner at the far-end. International journal of human-computer studies 72, 5 (2014), 477-487. ISBN: 1071-5819 Publisher: Elsevier.

[52] Lisa A Schur, Mason Ameri, and Douglas Kruse. 2020. Telework after COVID: a "silver lining" for workers with disabilities? fournal of occupational rehabilitation 30, 4 (2020), 521-536.

[53] Joseph Seering, Robert Kraut, and Laura Dabbish. 2017. Shaping Pro and Anti-Social Behavior on Twitch Through Moderation and Example-Setting. In
Proceedings of the 2017 ACM Conference on Computer Supported Cooperative Work and Social Computing. ACM, Portland Oregon USA, 111-125. https: //doi.org/10.1145/2998181.2998277

[54] Lucas M. Seuren, Joseph Wherton, Trisha Greenhalgh, and Sara E. Shaw. 2021. Whose turn is it anyway? Latency and the organization of turn-taking in videomediated interaction. Fournal of Pragmatics 172 (2021), 63 - 78. https://doi.org/ 10.1016/j.pragma.2020.11.005

[55] John C. Tang, Gina Venolia, and Kori M. Inkpen. 2016. Meerkat and Periscope: I Stream, You Stream, Apps Stream for Live Streams. In Proceedings of the 2016 CHI Conference on Human Factors in Computing Systems (CHI '16). Association for Computing Machinery, New York, NY, USA, 4770-4780. https://doi.org/10. $1145 / 2858036.2858374$

[56] Chris Weaver. 2004. Building highly-coordinated visualizations in improvise. In IEEE Symposium on Information Visualization. IEEE, 159-166.

[57] Donghee Yvette Wohn. 2019. Volunteer Moderators in Twitch Micro Communities: How They Get Involved, the Roles They Play, and the Emotional Labor They Experience. In Proceedings of the 2019 CHI Conference on Human Factors in Computing Systems. ACM, Glasgow Scotland Uk, 1-13. https: //doi.org/10.1145/3290605.3300390

[58] Donghee Yvette Wohn and Guo Freeman. 2020. Audience Management Practices of Live Streamers on Twitch. In ACM International Conference on Interactive Media Experiences. ACM, Cornella, Barcelona Spain, 106-116. https://doi.org/10. $1145 / 3391614.3393653$

[59] Qunfang Wu, Yisi Sang, and Yun Huang. 2019. Danmaku: A New Paradigm of Social Interaction via Online Videos. ACM Transactions on Social Computing 2, 2 (Oct. 2019), 1-24. https://doi.org/10.1145/3329485

[60] Sarita Yardi. 2006. The Role of the Backchannel in Collaborative Learning Environments. (2006), 7

[61] Zoom.us. 2021. Using in-meeting chat. https://support.zoom.us/hc/en-us/ articles/203650445-Using-in-meeting-chat 